\title{
A genetic algorithm for capital budgeting problem with fuzzy parameters
}

\begin{abstract}
When an organization utilizes modern technology in its manufacturing process, it needs to update and upgrade its facilities repetitively by efficient ways to stay with great productivity along with efficiency so. Capital Budgeting (CB) problem is one of the most important issues in decision makings about capital in the manufacturing management. Sometimes all variables and parameters are not necessarily deterministic and enough experiments are not available. Current study develops a chance constrained integer programming in the fuzzy environment for capital budgeting. Considering the complexity theory, a good answer could not be found in reasonable time, so that an intelligent Genetic Algorithm (GA) as a metaheuristic approach is provided to trace this problem with satisfying solutions. Thereupon, a fuzzy simulationbased genetic algorithm is provided for solving chance constrained integer programming model with fuzzy parameters.
\end{abstract}

Keyword: Capital budgeting; Goal programming; Fuzzy number; Genetic algorithm 